\title{
Single Degenerate Progenitors of Type Ia Supernovae
}

\section{Madelon Bours, Silvia Toonen, and Gijs Nelemans}

\author{
Department of Astrophysics, IMAPP, \\ Radboud University Nijmegen, The Netherlands \\ Contact: mbours@science.ru.nl
}

\begin{abstract}
There is a general agreement that Type Ia supernovae correspond to the thermonuclear runaway of a white dwarf (WD) in a compact binary. The details of these progenitor systems are still unclear. Using the population synthesis code SeBa and several assumption for the WD retention efficiency, we estimate the delay times and supernova rates for the single degenerate scenario.
\end{abstract}

Keywords. stars: supernovae, white dwarfs — binaries: close, symbiotic — accretion

\section{Single Degenerate Progenitors}

In this scenario a carbon/oxygen $(\mathrm{C} / \mathrm{O})$ white dwarf (WD) is in a binary with a (slightly) evolved star. This companion fills its Roche lobe and transfers hydrogen-rich matter from its outer layers onto the white dwarf. Here this accumulates with a certain retention efficiency $(\eta$, which represents the fraction of transferred mass that is retained by the WD), and burns into carbon. The white dwarf grows in mass, approaches $M_{C h}$, and its carbon ignites.

\section{White Dwarf Retention Efficiency}

Whether the matter that is transferred onto the $\mathrm{C} / \mathrm{O}$ white dwarf is actually retained by it depends on the mass transfer rate $\dot{M}_{H}$ and the white dwarf mass $M_{W D}$.

Medium $\dot{M}_{H}: \eta_{H}=1$, steady hydrogen burning

Low \& high $\dot{M}_{H}: \eta_{H}<1$, due to (strong) hydrogen novae \& white dwarf wind

The accumulation of helium onto the $\mathrm{C} / \mathrm{O}$ white dwarf is determined by the fraction of hydrogen-rich matter that is burned into helium-rich matter $\left(\eta_{H} \cdot \dot{M}_{H}\right)$. For He, a similar regions exists so that only for a limited range can helium novae be avoided. The total retention efficiency $\eta=\eta_{H} \cdot \eta_{H e}$ determines how much of the initially transferred matter is burned into carbon/oxygen-rich material so that the white dwarf can grow in mass. In the literature, different assumptions for determining $\eta$ are made (Hachisu et al. 2008, Nomoto et al. 2007, Prialnik \& Kovetz 1995), of which three are displayed in Figure 1.

\section{Delay Time Distributions}

A delay time distribution (DTD) is the rate of Type Ia supernovae (SNe Ia) as a function of time after a single starburst. The left side of figure 2 shows DTDs for three different retention efficiencies in combination with SeBa (Portegies \& Verbunt 1996). The numbers in the box show the corresponding integrated SNe Ia rate. Using different retention efficiencies does not significantly change the DTD shape, but effects the normalization. This can best be seen for the lowest $\eta$, producing no SNe Ia. 


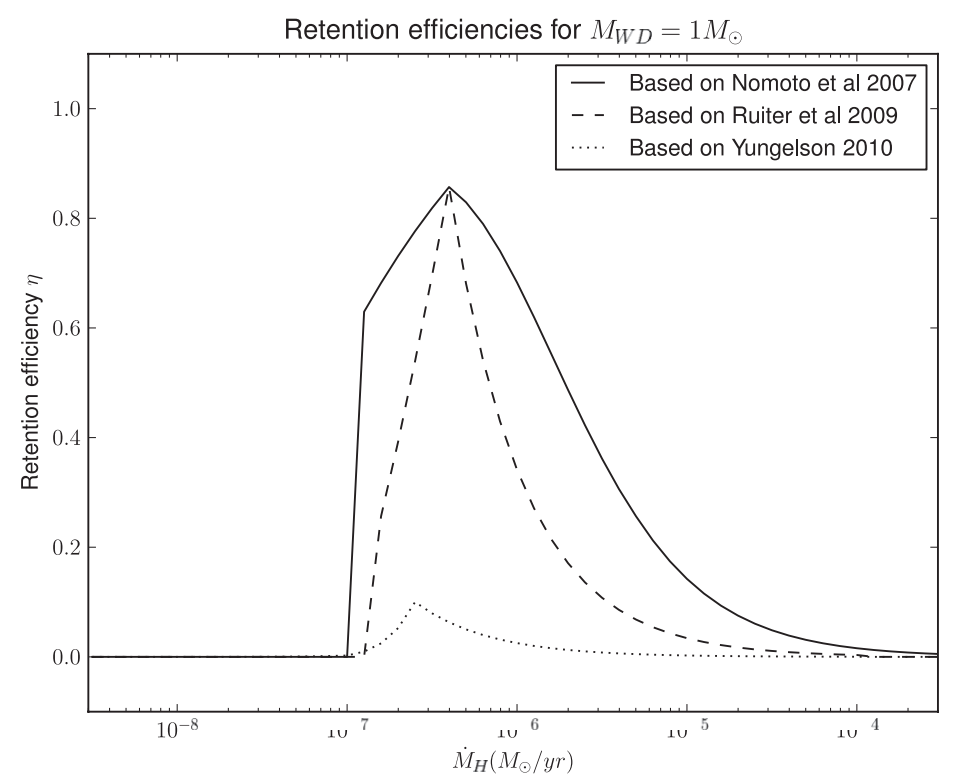

Figure 1. Total retention efficiencies $\eta=\eta_{H} \cdot \eta_{H e}$ as a function of hydrogen mass transfer rate, based on Nomoto et al. 2007, Ruiter et al. 2009 and Yungelson 2010.
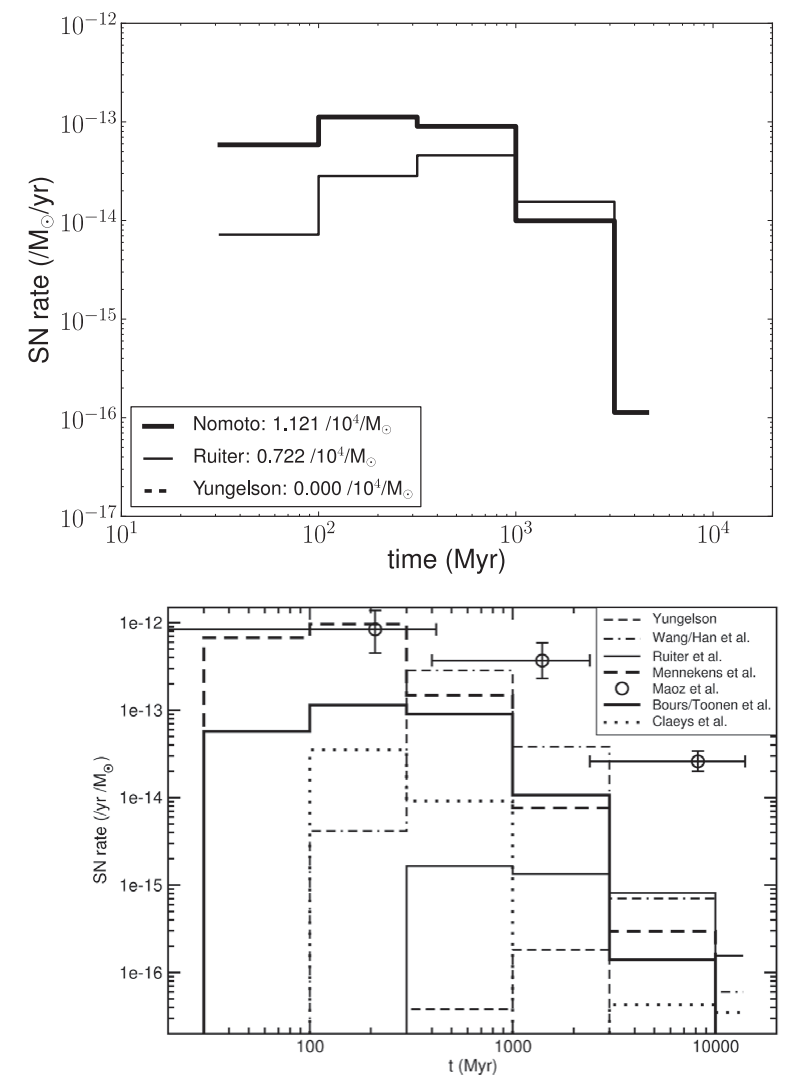

Figure 2. Top: DTDs and integrated rates from simulated populations with SeBa using different retention efficiencies. Bottom: DTDs from different research groups. Even with order of magnitude differences, the observational data are not reproduced. 
In $\mathrm{SeBa}$ the common envelope phase for these binaries is in general described by the $\gamma$ prescription (angular momentum conservation, Nelemans et al. 2000). A second possibility is the $\alpha$ prescription (energy conservation, Paczynski 1976). Changing from $\gamma$ to $\alpha$ evolution only slightly influences the DTD shape and integrated rates (Bours et al.).

The right side of Figure 2 shows the DTDs of several groups. The discrepancies can partly be explained by the use of a different retention efficiency. Partly the variation is caused by other assumptions in the various population synthesis codes used. Despite the efforts, with the current understanding of single degenerate progenitors, it is difficult to explain the observed SNe Ia time-integrated yield of $23 \cdot 10^{-4} \mathrm{M}_{\odot}^{-1}$ (Maoz et al. 2011).

\section{References}

Bours, M. C. P., Toonen, S. \& Nelemans, G. In prep.

Hachisu, I., Kato, M. \& Nomoto, K. 2008 ApJ, 679, 1390-1404

Maoz, D., Mannucci, F., Li, W., Filippenko, A.V., Della Valle, M. \& Panagia, N. 2011 MNRAS, $412,1508-1521$

Nelemans, G., Verbunt, F., Yungelson, L. R. \& Portegies Zwart, S. F. 2000 A\& A, 360, 1011-1018

Nomoto, K., Saio, H., Kato, M. \& Hachisu, I. 2007 ApJ, 663, 1269-1276

Paczynski, B. 1976 IAU Symposium, 75-+

Portegies, S. F. \& Verbunt, F. 1996, A\&AA, 309, 179

Prialnik, D. \& Kovetz, A. 1995 ApJ, 445, 789-810

Ruiter, A. J., Belczynski, K., \& Fryer, C. 2009 ApJ, 699, 2026-2036

Yungelson, L. R. 2010 Astron. Lett., 36, 780-787 